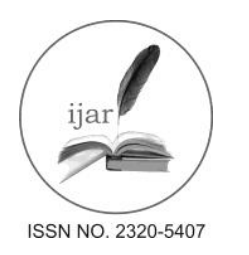

\author{
Journal homepage: http://www.journalijar.com

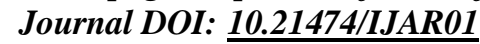

INTERNATIONAL JOURNAL

OF ADVANCED RESEARCH

RESEARCH ARTICLE

\title{
HEAT AND MASS TRANSFER ON MHD FLOW OVER AN INFINITE ROTATING OSCILLATING VERTICAL POROUS PLATE.
}

\author{
Dr .G. Kathyayani ${ }^{1}$, D. M. Praveen Babu ${ }^{2}$. \\ 1. Assistant Professor, Department of Applied Mathematics, Yogi Vemana University, Kadapa, A.P. \\ 2. Ph.D Scholar, Department of Applied Mathematics, Yogi Vemana University, Kadapa, A.P.
}

\section{Manuscript Info}

Manuscript History:

Received: 15 April 2016

Final Accepted: 29 May 2016

Published Online: June 2016

Key words:

Heat and mass transfer, MHD

flows, porous medium, unsteady

flows and visco-elastic fluids.

*Corresponding Author

Dr. G. Kathyayani.

\begin{abstract}
The unsteady MHD rotating flow of an incompressible Visco-elastic liquid of the Walter $B$ ' model with simultaneous heat and mass transfer near an infinite oscillating porous plate in slip flow regime under the influence of uniform transverse magnetic field has been discussed. The governing equations of the flow field are solved by a regular perturbation method for small elastic parameter. The expressions for the velocity, temperature, concentration have been derived analytically and also its behavior is computationally discussed with reference to different flow parameters with the help of graphs. The skin friction on the boundary, the heat flux in terms of the Nusselt number, and the rate of mass transfer in terms of the Sherwood number are also obtained and their behavior discussed.
\end{abstract}

Copy Right, IJAR, 2016,. All rights reserved.

\section{Introduction:-}

Flow of a viscous fluid in rotating channels is of considerable importance due to the occurrence of various natural phenomena and for its application in various technological situations which are governed by the action of Coriolis force. The broad subjects of oceanography, meteorology, atmospheric science and limnology all contain some important and essential features of rotating fluids. The viscous fluid flow problems in rotating medium under different conditions and configurations are investigated by many researchers in the past to analyze various aspects of the problem. The study of simultaneous effects of rotation and magnetic field on the fluid flow problems of a viscous incompressible electrically conducting fluid may find applications in the areas of geophysics, astrophysics and fluid engineering.

Engineering processes in which a fluid supports an exothermal chemical or nuclear reaction are very common today and the correct process design requires accurate correlation for the heat transfer coefficients at the boundary surfaces. Despite its increasing importance in technological and physical problems, the unsteady MHD free convection flows of dissipative fluids past an infinite plate have received much attention because of non-linearity of the governing equations. Saravana et.al.,[7] Mass Transfer effects on MHD Viscous flow past an impulsively started infinite vertical plate with constant mass flux. Abel and Veena [1] studied the Visco -Elasticity on the flow and heat transfer in a porous medium over a stretching sheet. All these studies deals with the studies concerning NonNewtonian flows and heat transfer in the absence of magnetic fields, but present years we find several industrial applications such as polymer technology and metallurgy where the magnetic field is applied in the Visco-Elastic fluid flow. Chemical reactions usually accompany a large amount of exothermic and endothermic reactions. These characteristics can be easily seen in a lot of industrial processes. Recently, it has been realized that it is not always permissible to neglect the convection effects in porous constructed chemical reactors. Kesavaiah et.al.,[5] effects of the chemical reaction and radiation absorption on an unsteady MHD convective heat and mass transfer flow past a semi-infinite vertical permeable moving plate embedded in a porous medium with heat source and suction. 
Modather et.al., [6] An analytical study of MHD heat and mass transfer oscillatory flow of a micropolar fluid over a vertical permeable plate in a porous medium.

Satyanarayana et.al., [8] viscous dissipation and thermal radiation effects on an unsteady MHD convection flow past a semi-infinite vertical permeable moving porous plate. Mass transfer effects on the flow past an exponentially accelerated vertical plate with constant heat flux was studied by Jha et al., [4]. Das et al., [2] have studied effects of mass transfer on flow past an impulsively started vertical infinite plate with constant heat flux and chemical reaction. Ezzat Magdy [3] have considered magneto hydrodynamic unsteady flow of non- Newtonian fluid past an infinite porous plate. Takhar et al.,[9] have studied radiation effects on MHD free convection flow of a radiating gas past a semi-infinite vertical plate. More recently Krishna and Prakash [12 ] discussed the unsteady flow of an incompressible viscous fluid in a rotating parallel plate channel bounded on one side by a porous bed under the influence of a uniform transverse magnetic field taking hall current into account. The effects of radiation and hall current on MHD free convection three dimensional flow in a vertical channel filled with a porous medium has been studied by Krishna and Chand basha [11]. Krishna [10] discussed the unsteady flow of an incompressible electrically conducting viscous fluid in a rotating porous media, with a variable pressure gradient and in the presence of hall current.

The objective of the present paper is to analyze the heat and mass transfer effects on MHD Visco-elastic rotating flow past an oscillating porous plate in slip flow regime under the influence of uniform transverse magnetic field with heat source and chemical reaction.

\section{Formulation and Solution of the Problem:-}

We consider the unsteady MHD rotating flow of an incompressible visco-elastic liquid of Walter's $B$ ' model through porous medium with simultaneous heat and mass transfer near an oscillating infinite porous plate in slip flow regime with heat source and chemical reaction under the influence of a uniform transverse magnetic field of strength $\mathrm{H}_{\mathrm{o}}$. The z-axis is taken along the plate in vertical direction, and $x$-axis is perpendicular to it. A uniform magnetic field of strength $B_{0}$ is applied in the direction of z-axis. Let $u$ and $\mathrm{v}$ be the velocity components in $\mathrm{x}$ - and y-directions, respectively. If the plate is extended to infinite length, then all the physical variables in the problem are functions of $\mathrm{z}$ and $t$ alone. Initially, the plate and fluid are at rest, and then the plate is set to an oscillatory motion. The Reynolds number is assumed to be very small, and the induced magnetic field due to the flow is neglected with respect to the applied magnetic field. The pressure in the fluid is assumed to be constant. If $\mathrm{w}_{0}$ represents the suction or injection velocity at the plate. The physical configuration of the problem is as shown in Figure.1.

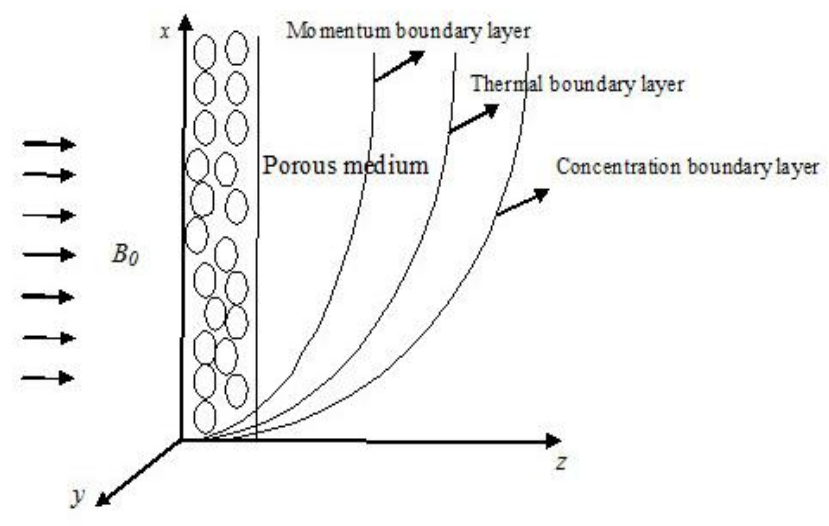

Figure 1: Physical configuration of the problem

Then governing equations for the unsteady MHD rotating flow of an incompressible visco-elastic liquid of the Walter $B^{\prime}$ model with simultaneous heat and mass transfer near an oscillating porous plate in slip flow regime are,

$$
\begin{gathered}
\frac{\partial w}{\partial z}=0 \\
\frac{\partial u}{\partial t}-w_{0} \frac{\partial u}{\partial z}-2 \Omega v=v \frac{\partial^{2} u}{\partial z^{2}}-\frac{K_{0}}{\rho}\left(\frac{\partial^{3} u}{\partial z^{2} \partial t}+v_{0} \frac{\partial^{3} u}{\partial z^{3}}\right)-\frac{\sigma B_{0}^{2}}{\rho} u-\frac{v}{k} u+g \beta\left(T-T_{\infty}\right)+g \beta *\left(C-C_{\infty}\right)
\end{gathered}
$$




$$
\begin{gathered}
\frac{\partial v}{\partial t}-w_{0} \frac{\partial v}{\partial z}+2 \Omega u=v \frac{\partial^{2} v}{\partial z^{2}}-\frac{K_{0}}{\rho}\left(\frac{\partial^{3} v}{\partial z^{2} \partial t}+v_{0} \frac{\partial^{3} v}{\partial z^{3}}\right)-\frac{\sigma B_{0}^{2}}{\rho} v-\frac{v}{k} v \\
\frac{\partial T}{\partial t}-w_{0} \frac{\partial T}{\partial z}=\alpha \frac{\partial^{2} T}{\partial z^{2}}-S\left(T-T_{\infty}\right) \\
\frac{\partial C}{\partial t}-w_{0} \frac{\partial C}{\partial z}=D \frac{\partial^{2} C}{\partial z^{2}}-K_{1}\left(C-C_{\infty}\right)
\end{gathered}
$$

Combining the equations (2.2) and (2.3), let $q=u+i v$,

$$
\frac{\partial q}{\partial t}-w_{0} \frac{\partial q}{\partial z}+2 i \Omega q=v \frac{\partial^{2} q}{\partial z^{2}}-\frac{K_{0}}{\rho}\left(\frac{\partial^{3} q}{\partial z^{2} \partial t}+w_{0} \frac{\partial^{3} q}{\partial z^{3}}\right)-\frac{\sigma B_{0}^{2}}{\rho} q-\frac{v}{K} q+g \beta\left(T-T_{\infty}\right)+g \beta *\left(C-C_{\infty}\right)
$$

The first order velocity slip boundary conditions of the problem when the plate executes linear harmonic oscillations in its own plane are given by

$$
\begin{aligned}
q=U_{0} e^{i \omega t}+L_{1} \frac{\partial q}{\partial z}, T & =T_{w}, C=C_{w} \quad \text { at } \quad z=0 \\
q & =0, T \rightarrow T_{\infty}, C \rightarrow C_{\infty} \quad \text { at } \quad z \rightarrow \infty
\end{aligned}
$$

Where, $L_{1}=\left(2-m_{1}\right)\left(L / m_{1}\right), L=\mu(\pi / 2 p \rho)^{1 / 2}$ is the mean free path, and $m_{1}$ is Maxwell's reflection coefficient.

On introducing the following non-dimensional quantities

$$
\begin{gathered}
u^{*}=\frac{u}{U_{0}}, v^{*}=\frac{v}{U_{0}}, t^{*}=\frac{t U_{0}^{2}}{v}, z^{*}=\frac{z U_{0}}{v}, \omega^{*}=\frac{v}{U_{0}^{2}} \omega, \\
w_{0}^{*}=\frac{w_{0}}{U_{0}}, \theta=\frac{T-T_{\infty}}{T_{w}-T_{\infty}}, C^{*}=\frac{C-C_{\infty}}{C_{w}-C_{\infty}}
\end{gathered}
$$

Making use of non-dimensional variables, the governing equations reduces to (Dropping asterisks)

$$
\begin{aligned}
& \frac{\partial \boldsymbol{q}}{\partial t}-w_{\mathrm{o}} \frac{\partial \boldsymbol{q}}{\partial z}+2 i E \boldsymbol{q}=\frac{\partial^{2} \boldsymbol{q}}{\partial z^{2}}-\boldsymbol{R c}\left(\frac{\partial^{3} \boldsymbol{q}}{\partial z^{2} \partial t}+v_{\mathrm{o}} \frac{\partial^{3} \boldsymbol{q}}{\partial z^{3}}\right)-\left(\boldsymbol{M}^{2}+\frac{1}{K}\right) \boldsymbol{q}+\boldsymbol{G r} \boldsymbol{\theta}+\boldsymbol{G} \boldsymbol{m} \boldsymbol{C} \\
& \operatorname{Pr} \frac{\partial \theta}{\partial t}-w_{0} \frac{\partial \theta}{\partial z}=\frac{\partial^{2} \theta}{\partial z^{2}}-\operatorname{Pr} S \theta \\
& S c \frac{\partial C}{\partial t}-w_{0} \frac{\partial C}{\partial z}=\frac{\partial^{2} C}{\partial z^{2}}-K c S c C
\end{aligned}
$$

Corresponding boundary conditions are,

$$
\begin{gathered}
q=e^{i \omega t}+R \frac{\partial q}{\partial z}, \quad \theta=1, \quad C=1 \quad \text { at } \quad z=0 \\
q=0, \theta=0, C=0 \quad \text { at } \quad z \rightarrow \infty
\end{gathered}
$$

Equation (2.9) is of third order, and two boundary conditions are available. Due to inadequate boundary condition, a perturbation method has been applied with $R c<1$ as the perturbation parameter. This assumption is quite consistent as the model under consideration is valid only for slightly elastic fluid. We have considered the following,

$$
\begin{aligned}
& q=q_{0}+R c q_{1}+O(R c)^{2} \\
& \theta=\theta_{0}+R c \theta_{1}+O(R c)^{2} \\
& C=C_{0}+R c C_{1}+O(R c)^{2}
\end{aligned}
$$

Substituting (2.14) to (2.16) in (2.9) - (2.11) and equating like powers of $R c$, we get the following. 
Zeroth order:-

$$
\begin{gathered}
\frac{\partial q_{0}}{\partial t}-w_{0} \frac{\partial q_{0}}{\partial z}=\frac{\partial^{2} q_{0}}{\partial z^{2}}-\left(2 i E+M^{2}+\frac{1}{K}\right) q_{0}+G r \theta_{0}+G m C_{0} \\
\operatorname{Pr} \frac{\partial \theta_{0}}{\partial t}-w_{0} \frac{\partial \theta_{0}}{\partial z}=\frac{\partial^{2} \theta_{0}}{\partial z^{2}}-\operatorname{Pr} S \theta_{0} \\
S c \frac{\partial C_{0}}{\partial t}-w_{0} \frac{\partial C_{0}}{\partial z}=\frac{\partial^{2} C_{0}}{\partial z^{2}}-K c S c C_{0}
\end{gathered}
$$

First order:-

$$
\begin{gathered}
\frac{\partial q_{1}}{\partial t}-w_{0} \frac{\partial q_{1}}{\partial z}=\frac{\partial^{2} q_{1}}{\partial z^{2}}-\left(\frac{\partial^{3} q_{0}}{\partial z^{2} \partial t}+v_{0} \frac{\partial^{3} q_{1}}{\partial z^{3}}\right)-\left(2 i E+M^{2}+\frac{1}{K}\right) q_{1}+G r \theta_{1}+G m C_{1} \\
\operatorname{Pr} \frac{\partial \theta_{1}}{\partial t}-w_{0} \frac{\partial \theta_{1}}{\partial z}=\frac{\partial^{2} \theta_{1}}{\partial z^{2}}-\operatorname{Pr} S \theta_{1} \\
S c \frac{\partial C_{1}}{\partial t}-w_{0} \frac{\partial C_{1}}{\partial z}=\frac{\partial^{2} C_{1}}{\partial z^{2}}-K c S c C_{1}
\end{gathered}
$$

The corresponding boundary conditions

$$
\begin{aligned}
& q_{0}=e^{i \omega t}+R \frac{\partial q_{0}}{\partial z}, q_{1}=1, \theta_{0}=1, \theta_{1}=1, C_{0}=1, C_{1}=1 \quad \text { at } \quad z=0 \\
& q_{0}=0, q_{1}=0, \theta_{0}=0, \theta_{1}=0, C_{0}=0, C_{1}=0 \quad \text { at } z \rightarrow \infty
\end{aligned}
$$

In order to reduce the system of partial differential equations (2.20)-(2.22) to a system of ordinary differential equations, we further introduce

$$
\begin{gathered}
q_{0}(z, t)=q_{00}(z)+q_{01}(z) e^{i \omega t} \\
q_{1}(z, t)=q_{10}(z)+q_{11}(z) e^{i \omega t} \\
\theta_{0}(z, t)=\theta_{00}(z)+q_{01}(z) e^{i \omega t} \\
\theta_{1}(z, t)=\theta_{10}(z)+q_{11}(z) e^{i \omega t} \\
C_{0}(z, t)=C_{00}(z)+C_{01}(z) e^{i \omega t} \\
C_{1}(z, t)=C_{10}(z)+C_{11}(z) e^{i \omega t}
\end{gathered}
$$

Substituting (2.25) to (2.30) into (2.20) - (2.22) and equating the harmonic and non-harmonic terms, we obtain the velocity, temperature and concentration of the flow field are

\section{Skin friction:-}

$$
\theta=e^{-a_{1} z}, C=e^{-a_{2} z}
$$

$$
\begin{aligned}
& \text { The skin friction at the plate is given by } \\
& \tau=\frac{\tau_{x y}}{U_{0}^{2}}=\frac{\partial u}{\partial z}-\left.R c\left(\frac{\partial^{2} q}{\partial t \partial z}+w_{0} \frac{\partial^{2} q}{\partial z^{2}}\right)\right|_{z=0}, \text { Where } \tau_{x y}=\frac{\partial u}{\partial z}-\frac{K_{0}}{\rho}\left(\frac{\partial^{2} q}{\partial t \partial z}+w_{0} \frac{\partial^{2} q}{\partial z^{2}}\right) \\
& \tau=-a_{1} m_{1}-a_{2} m_{2}-a_{3} m_{3}+a_{1} m_{1}^{2}+a_{2} m_{2}^{2}+a_{3} m_{3}^{2}-\left(a_{4} m_{4}(1+i \omega)-a_{4} m_{4}^{2}\right) e^{i \omega t} \\
& +R c\left(\left(-a_{5} m_{1}-a_{6} m_{2}-a_{7} m_{3}+a_{5} m_{1}^{2}+a_{6} m_{2}^{2}+a_{7} m_{3}^{2}\right)-\left(a_{8} m_{4}(1+i \omega)-a_{8} m_{4}^{2}\right) e^{i \omega t}\right)
\end{aligned}
$$

\section{Nusselt number:-}

The rate of heat transfer, that is, the heat flux at the plate $\mathrm{z}=0$ in terms of the Nusselt number, is given by

\section{Sherwood number:-}

$$
N u=-\left.\frac{\partial \theta}{\partial z}\right|_{z=0}=m_{1}
$$


The rate of mass transfer at the plate $\mathrm{z}=0$ in terms of the Sherwood number is given by

$$
S h=-\left.\frac{\partial C}{\partial z}\right|_{z=0}=m_{2}
$$

\section{Results and Discussions:-}

We considered the unsteady flow of an incompressible Visco-elastic liquid of the Walter $B$ ' model with simultaneous heat and mass transfer near an oscillating porous plate in slip flow regime under the influence of uniform transverse magnetic field. The effects of the non-dimensional parameters on the governing flow such as Hartmann number $\mathrm{M}$, the porosity parameter $K$, Prandtl number Pr; the elastic parameter Rc, the chemical reaction parameter Kc, the heat source parameter S; thermal Grashof number Gr, the mass Grashof number Gm, the Scmidt number Sc; the suction parameter $\mathrm{w}_{0}$, rarefaction parameter $\mathrm{R}, \mathrm{E}$ the rotation parameter and the frequency of oscillation $\omega$ on the velocity field have been studied analytically and presented with the help of Figures (2-13). The effects of the governing flow parameters on the temperature and concentration distributions have been presented in Figures (14-20), respectively. Further, the effects of the flow parameters on the skin friction, Nusselt number, and rate of mass transfer have been discussed with the help of Tables (1-3). For numerical computation, the values of Gr are taken positive. This indicates that the study has been carried out under the influence of the cooling of the plate. The interesting aspect of the problem is to study the combined effect of the flow parameters with that of the first order velocity slip boundary condition when the plate executes linear harmonic oscillation in its own plane. For computational purpose we are fixing the values $\mathrm{M}=2, \mathrm{~K}=1, \mathrm{Pr}=0.71, \mathrm{Rc}=0.5, \mathrm{Kc}=1, \mathrm{~S}=1, \mathrm{Gr}=5, \mathrm{Gm}=10, \mathrm{Sc}=0.22$, $\mathrm{w}_{0}=2, \mathrm{R}=2, \omega=\pi / 4, E=1$ and $t=0.1$.

Figures (2-7) shown the effect of Hartmann number M, the permeability parameter K and the Prandtl number Pr on velocity profile. It is observed that the increase in the Prandtl number as well as permeability parameter decreases both the velocity components of the flow field, whereas increase in Hartmann number increases it. Since Prandtl number is the ratio of kinematic viscosity to thermal diffusivity, so as Pr increases, the kinematic viscosity of the fluid dominates the thermal diffusivity of the fluid which leads to decreasing the velocity of the flow field. The application of transverse magnetic field sets up the Lorentz force, which enhances the fluid velocity.

Figure (8-13) depicts the effect of the thermal Grashof number Gr, the mass Grashof number Gm, and the Schmidt number Sc, on the velocity components $\mathrm{u}$ and $\mathrm{v}$. It is observed that, the velocity component $\mathrm{u}$ decreases and $\mathrm{v}$ increases with increasing thermal Grashof number Gr. Moreover, the velocity component $u$ enhances with Gm and reduces with $\mathrm{Sc}$, while the velocity component $\mathrm{v}$ first reduces and then sharply increases with increasing the mass Grashof number Gm or Schmidt number Sc. For the heavier species, that is, with increasing Sc, the velocity decreases.

Figures (14-16) showed the effect of the Prandtl number Pr, heat source parameter $\mathrm{S}$, and suction parameter $\left(\mathrm{w}_{0}\right)$ on the temperature of the flow field. We noted that the temperature of the flow field diminishes as the Prandtl number increases. This is consistent with the fact that the thermal boundary layer thickness decreases with increasing Prandtl number. With increasing heat source parameter reduces the temperature of the flow field. This may happen due the elastic property of the fluid. It is observed that temperature of the flow field diminishes as the suction parameter increases.

Figures (17-20) depict the effect of chemical reaction parameter Kc, the Schmidt number, Sc and suction parameter $\left(\mathrm{w}_{0}\right)$ on concentration distribution. The concentration distribution decreases at all points of the flow field with the increase in the Schmidt number Sc. This shows that the heavier diffusing species have a greater retarding effect on the concentration distribution of the flow field. It is observed that a destructive reaction $(\mathrm{Kc}>0)$ reduces the concentration distribution, whereas a generative reaction $(\mathrm{Kc}=0)$ enhances it. Also, it is observed that presence of suction parameter diminishes the concentration distribution.

The skin friction is an significant phenomenon which characterizes the frictional drag force at the solid surface. From Table 1, it is observed that the skin friction increases with the increase in all the forcing forces, but it is interesting to note that the skin friction decreases with the increase in M, Gm, $\omega$ and E. From Table 2, it is to note that all the entries are positive. It is seen that the Prandtl number (Pr), heat source parameter (S) and suction parameter $\left(\mathrm{w}_{0}\right)$ increase the rate of heat transfer (Nusselt number $\mathrm{Nu}$ ) at the surface of the plate. From Table 3 it is to note that all the entries are positive. It is observed that Schmidt number $(\mathrm{Sc})$, chemical reaction parameter (Kc), and suction parameter $\left(\mathrm{w}_{0}\right)$ increase the rate of mass transfer at the surface of the plate. 


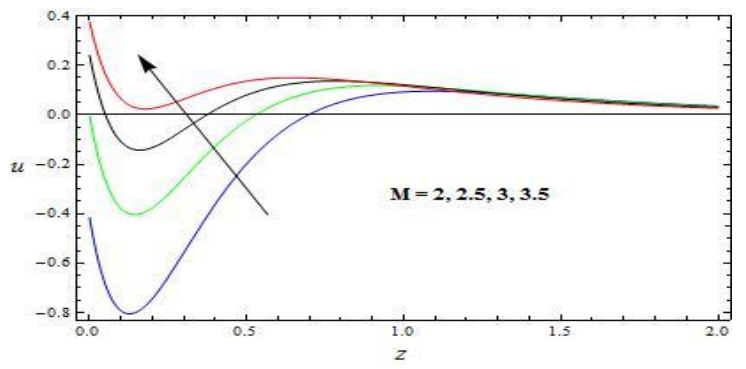

Figure 2:- The velocity Profile for u with M

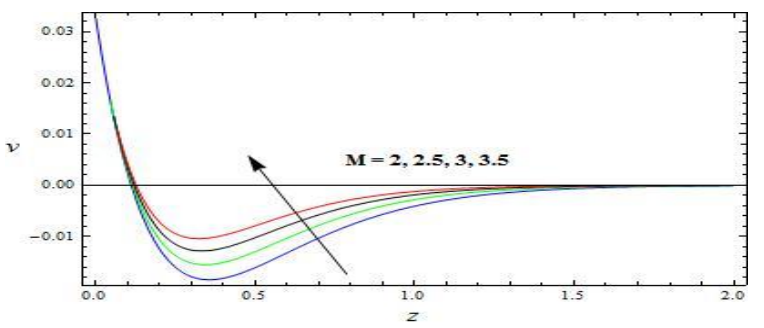

Figure 3:- The velocity Profile for v with M

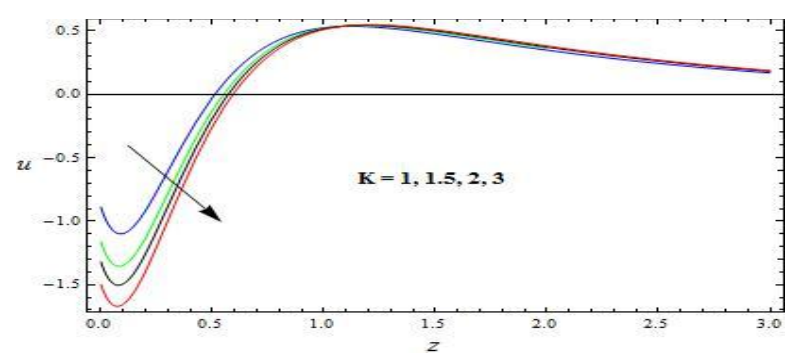

Figure 4:- The velocity Profile for u with K

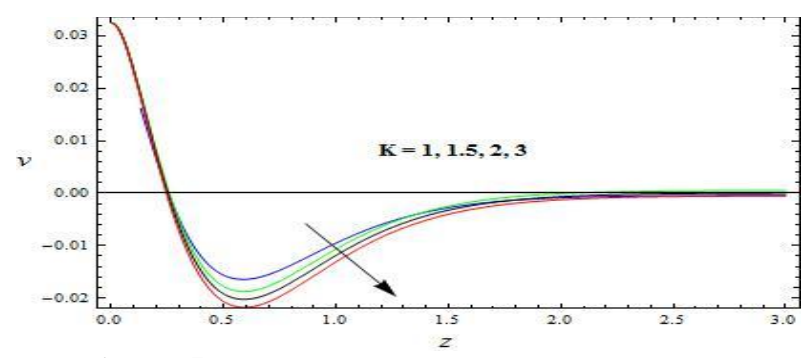

Figure 5:- The velocity Profile for v with K

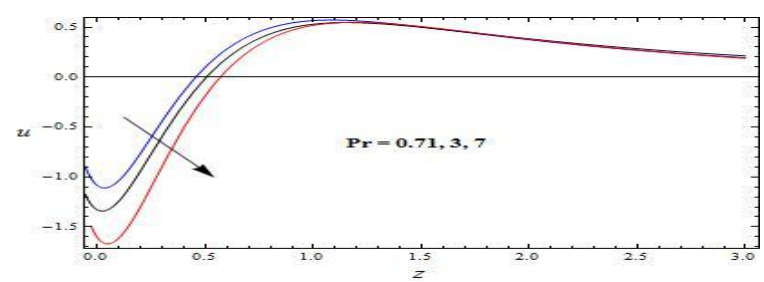

Figure 6. The velocity Profile for $\mathrm{u}$ with $\mathrm{Pr}$

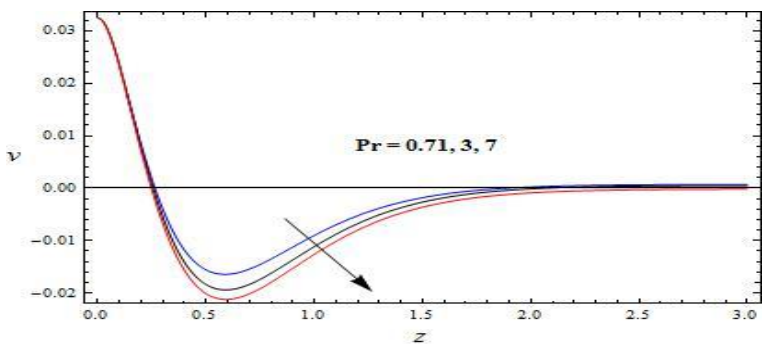

Figure 7:- The velocity Profile for v with Pr.

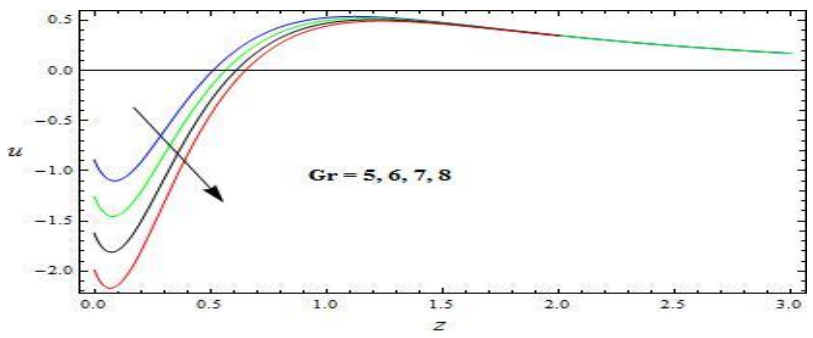

Figure 8:- The velocity Profile for u with $\mathrm{Gr}$

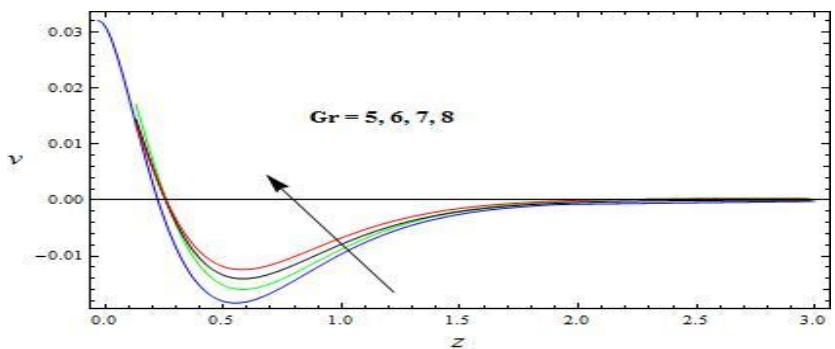

Figure 9:- The velocity Profile for v with Gr

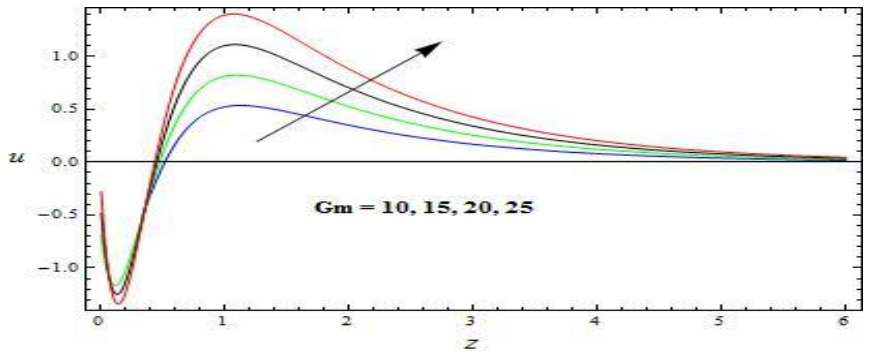

Figure 10:- The velocity Profile for $\mathrm{u}$ with Gm

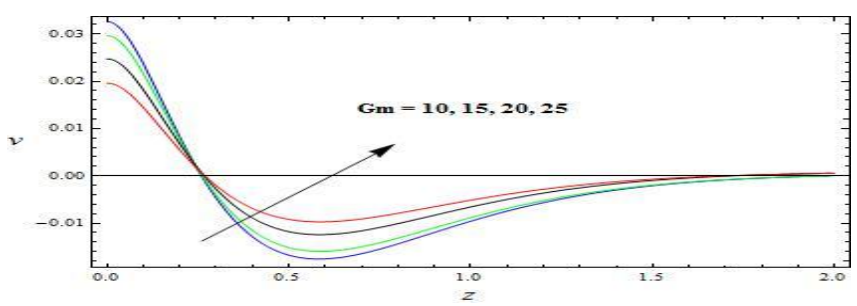

Figure 11. The velocity Profile for v with Gm 


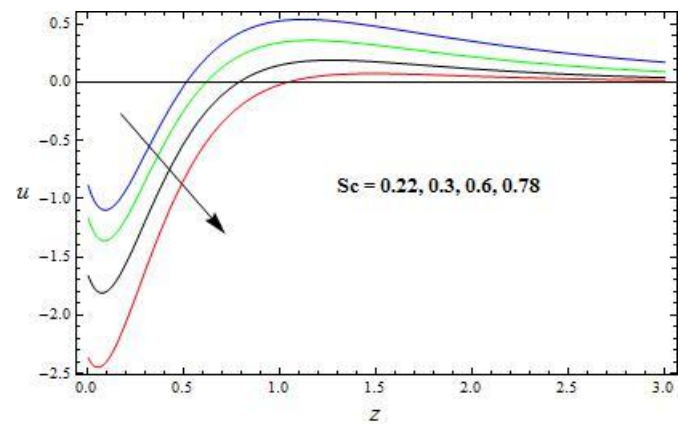

Figure 12:- The velocity Profile for $u$ with Sc

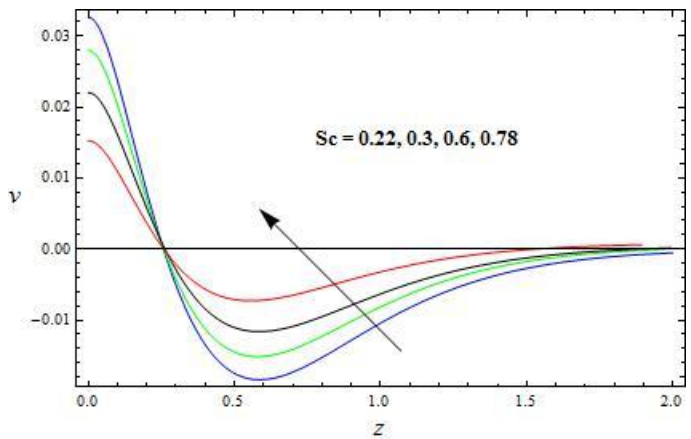

Figure 13:- The velocity Profile for v with Sc

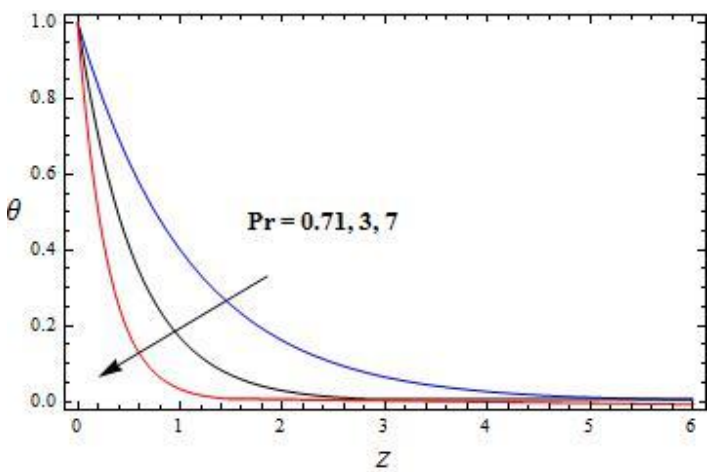

Figure 14:- Temperature Profiles for Pr

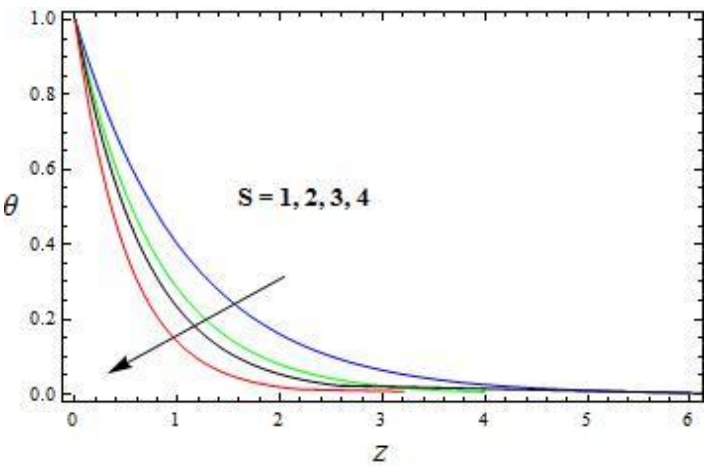

Figure 15:- Temperature Profiles for $S$

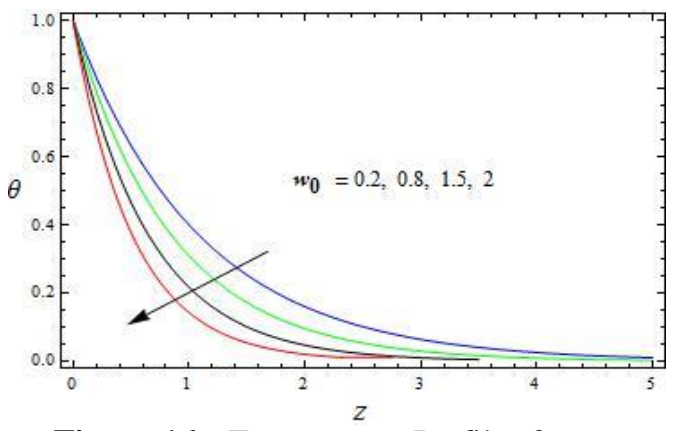

Figure 16:- Temperature Profiles for $\mathrm{w}_{0}$

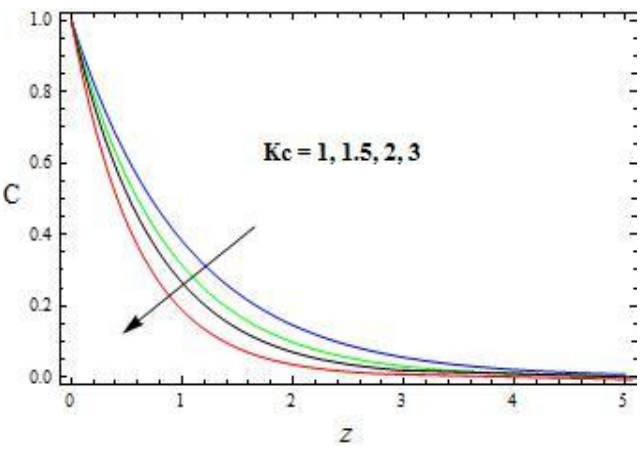

Figure 17:- Concentration Profiles for Kc

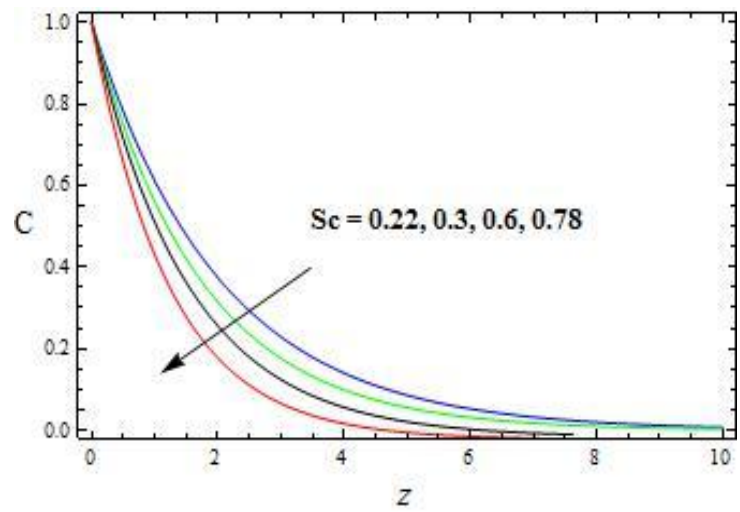

Figure 18:- Concentration Profiles for Sc

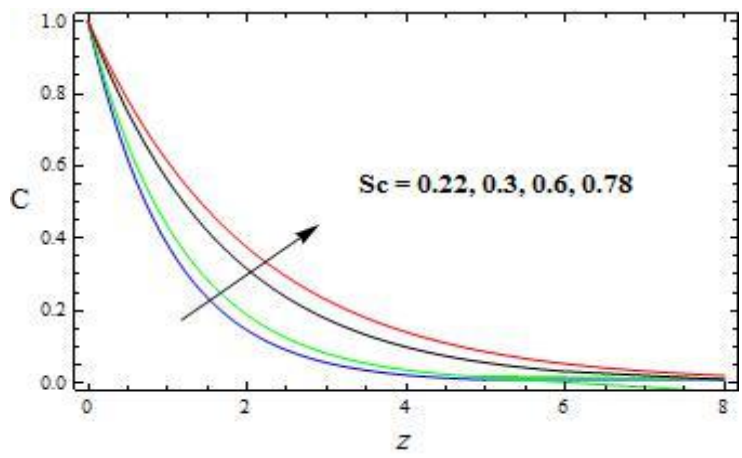

Figure 19:- Concentration Profiles for Sc 


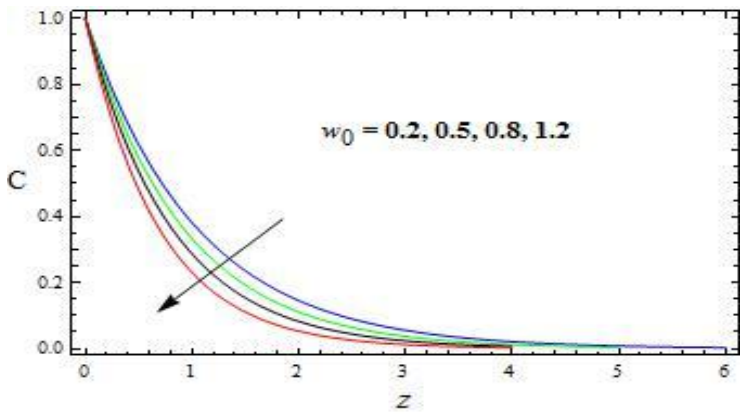

Figure 20:- Concentration Profiles for $\mathrm{w}_{0}$

\begin{tabular}{|c|c|c|c|c|c|c|c|c|c|c|c|c|c|}
\hline $\mathrm{M}$ & $\mathrm{K}$ & $w_{0}$ & $\mathrm{R}$ & $\mathrm{Pr}$ & $\mathrm{Gr}$ & $\mathrm{Gm}$ & $\mathrm{Sc}$ & $\omega$ & $\mathrm{S}$ & $\mathrm{Kc}$ & $\mathrm{Rc}$ & $\boldsymbol{E}$ & $\tau$ \\
\hline $\mathbf{2}$ & $\mathbf{1}$ & $\mathbf{2}$ & $\mathbf{0 . 2}$ & $\mathbf{0 . 7 1}$ & $\mathbf{5}$ & $\mathbf{1 0}$ & $\mathbf{0 . 2 2}$ & $\pi / 4$ & 1 & $\mathbf{1}$ & $\mathbf{1}$ & $\mathbf{1}$ & 16.93600 \\
\hline $\mathbf{4}$ & 1 & 2 & 0.2 & 0.71 & 5 & 10 & 0.22 & $\pi / 4$ & 1 & 1 & 1 & 1 & 8.165483 \\
\hline $\mathbf{6}$ & 1 & 2 & 0.2 & 0.71 & 5 & 10 & 0.22 & $\pi / 4$ & 1 & 1 & 1 & 1 & 5.039669 \\
\hline 2 & $\mathbf{2}$ & 2 & 0.2 & 0.71 & 5 & 10 & 0.22 & $\pi / 4$ & 1 & 1 & 1 & 1 & 20.30430 \\
\hline 2 & $\mathbf{3}$ & 2 & 0.2 & 0.71 & 5 & 10 & 0.22 & $\pi / 4$ & 1 & 1 & 1 & 1 & 21.64770 \\
\hline 2 & 1 & $\mathbf{2 . 2}$ & 0.2 & 0.71 & 5 & 10 & 0.22 & $\pi / 4$ & 1 & 1 & 1 & 1 & 33.61497 \\
\hline 2 & 1 & $\mathbf{2 . 5}$ & 0.2 & 0.71 & 5 & 10 & 0.22 & $\pi / 4$ & 1 & 1 & 1 & 1 & 75.33262 \\
\hline 2 & 1 & 2 & $\mathbf{0 . 5}$ & 0.71 & 5 & 10 & 0.22 & $\pi / 4$ & 1 & 1 & 1 & 1 & 21.07570 \\
\hline 2 & 1 & 2 & $\mathbf{0 . 8}$ & 0.71 & 5 & 10 & 0.22 & $\pi / 4$ & 1 & 1 & 1 & 1 & 22.77120 \\
\hline 2 & 1 & 2 & 0.2 & $\mathbf{7}$ & 5 & 10 & 0.22 & $\pi / 4$ & 1 & 1 & 1 & 1 & 195.3193 \\
\hline 2 & 1 & 2 & 0.2 & 0.71 & $\mathbf{8}$ & 10 & 0.22 & $\pi / 4$ & 1 & 1 & 1 & 1 & 31.04973 \\
\hline 2 & 1 & 2 & 0.2 & 0.71 & $\mathbf{1 0}$ & 10 & 0.22 & $\pi / 4$ & 1 & 1 & 1 & 1 & 40.47114 \\
\hline 2 & 1 & 2 & 0.2 & 0.71 & 5 & $\mathbf{1 5}$ & 0.22 & $\pi / 4$ & 1 & 1 & 1 & 1 & 11.88353 \\
\hline 2 & 1 & 2 & 0.2 & 0.71 & 5 & $\mathbf{2 0}$ & 0.22 & $\pi / 4$ & 1 & 1 & 1 & 1 & 8.402142 \\
\hline 2 & 1 & 2 & 0.2 & 0.71 & 5 & 10 & $\mathbf{0 . 6}$ & $\pi / 4$ & 1 & 1 & 1 & 1 & 50.72582 \\
\hline 2 & 1 & 2 & 0.2 & 0.71 & 5 & 10 & $\mathbf{0 . 7 8}$ & $\pi / 4$ & 1 & 1 & 1 & 1 & 96.21521 \\
\hline 2 & 1 & 2 & 0.2 & 0.71 & 5 & 10 & 0.22 & $\pi / 3$ & 1 & 1 & 1 & 1 & 16.22817 \\
\hline 2 & 1 & 2 & 0.2 & 0.71 & 5 & 10 & 0.22 & $\pi / 2$ & 1 & 1 & 1 & 1 & 14.79243 \\
\hline 2 & 1 & 2 & 0.2 & 0.71 & 5 & 10 & 0.22 & $\pi / 4$ & 1.5 & 1 & 1 & 1 & 30.21766 \\
\hline 2 & 1 & 2 & 0.2 & 0.71 & 5 & 10 & 0.22 & $\pi / 4$ & 2 & 1 & 1 & 1 & 46.9786 \\
\hline 2 & 1 & 2 & 0.2 & 0.71 & 5 & 10 & 0.22 & $\pi / 4$ & 1 & 2 & 1 & 1 & 20.07074 \\
\hline 2 & 1 & 2 & 0.2 & 0.71 & 5 & 10 & 0.22 & $\pi / 4$ & 1 & 3 & 1 & 1 & 23.69089 \\
\hline 2 & 1 & 2 & 0.2 & 0.71 & 5 & 10 & 0.22 & $\pi / 4$ & 1 & 1 & 1.2 & 1 & 23.93083 \\
\hline 2 & 1 & 2 & 0.2 & 0.71 & 5 & 10 & 0.22 & $\pi / 4$ & 1 & 1 & 1.5 & 1 & 34.42666 \\
\hline 2 & 1 & 2 & 0.2 & 0.71 & 5 & 10 & 0.22 & $\pi / 4$ & 1 & 1 & 1 & 2 & 10.01081 \\
\hline 2 & 1 & 2 & 0.2 & 0.71 & 5 & 10 & 0.22 & $\pi / 4$ & 1 & 1 & 1 & 3 & 5.497744 \\
\hline
\end{tabular}

Table. 1:- Skin Friction

\begin{tabular}{|c|c|c|c|}
\hline $\mathrm{S}$ & $\mathrm{Pr}$ & $w_{0}$ & $\mathrm{Nu}$ \\
\hline 0.5 & 0.71 & 0.2 & 0.671034 \\
\hline 1 & 0.71 & 0.2 & 0.916600 \\
\hline 1.5 & 0.71 & 0.2 & 1.105427 \\
\hline 0.5 & 3 & 0.2 & 1.560952 \\
\hline 0.5 & 7 & 0.2 & 2.697498 \\
\hline 0.5 & 0.71 & 0.5 & 0.799196 \\
\hline 0.5 & 0.71 & 0.8 & 0.944042 \\
\hline
\end{tabular}

Table 2:- Nusselt number

\begin{tabular}{|c|c|c|c|}
\hline Sc & $w_{0}$ & Kc & Sh \\
\hline 0.22 & 0.2 & 1 & 0.491557 \\
\hline 0.3 & 0.2 & 1 & 0.578543 \\
\hline 0.6 & 0.2 & 1 & 0.836916 \\
\hline 0.78 & 0.2 & 1 & 0.964613 \\
\hline 0.22 & 0.5 & 1 & 0.527255 \\
\hline 0.22 & 0.8 & 1 & 0.565225 \\
\hline 0.22 & 0.2 & 2 & 0.685689 \\
\hline 0.22 & 0.2 & 3 & 0.834701 \\
\hline
\end{tabular}

Table 3:- Sherwood number 


\section{Conclusions:-}

A theoretical study of unsteady MHD incompressible visco-elastic liquid of Walter's model with heat and mass transfer near an oscillating infinite porous plate in slip flow regime under the influence of a uniform transverse magnetic field is considered. Some of the important findings are given in the following.

1. The magnitudes of the velocity components decrease with increasing $\operatorname{Pr}$ and $\mathrm{K}$, whereas presence of magnetic field and rotation increases it.

2. The resultant velocity of the flow field decreases suddenly near the plate due to the presence of elastic parameter.

3. The velocity of the flow field decreases due to the increase in the thermal Grashof number.

4. The resultant velocity experiences retardation and then increases with increasing rarefaction parameter $\mathrm{R}$, whereas the reverse effect is observed due the presence of the frequency of oscillation.

5. Thermal boundary layer thickness decreases with increasing the Prandtl number Pr.

6. Heavier diffusing species have a greater retarding effect on the concentration distribution.

\section{References:-}

1. Abel.S, and Veena.P.H (1998), "Visco-Elastic Fluid Flow and Heat Transfer in Porous Medium over a Stretching Sheet," International Journal of Non-Linear Mechanics, 33, pp. 531-540.

2. Das.U.N, Deka.R.K, and Soundalgekar.V.M (1994), "Effects of mass transfer on flow past an impulsively started vertical infinite vertical plate with constant heat flux and chemical reaction," Forschung in Ingenieurwesen, 60, 284-287.

3. Ezzat Magdy .A (1994). Magnetohydrodynamic unsteady flow of non- Newtonian fluid past an infinite porous plate. Indian J. pure appl. math. 25(6), 655 - 664.

4. Jha.B.K, Prasad.R, and Rai.S (1991), "Mass transfer effects on the flow past an exponentially accelerated vertical plate with constant heat flux," Astrophysics and Space Science, 181, 125- 134.

5. Kesavaiah.D.Ch, Satyanarayana.P.V, and Venkataramana.S (2011), "Effects of the chemical reaction and radiation absorption on an unsteady MHD convective heat and mass transfer flow past a semi-infinite vertical permeable moving plate embedded in a porous medium with heat source and suction," Int. J. of Appl. Math and Mech., 7 (1), 52-69.

6. Modather.M, Rashad.A.M, and Chamkha.A.J (2009), "An analytical study of MHD heat and mass transfer oscillatory flow of a micropolar fluid over a vertical permeable plate in a porous medium," Turkish J. Eng. Env. Sci. 33, 1-13.

7. Saravana.R, Sreekanth.S, Sreenadh.S, Hemadri Reddy.R (2011), "Mass Transfer effects on MHD Viscous flow past an impulsively started infinite vertical plate with constant mass flux," Advances in Applied Science Research, 2 (1), 221-229.

8. SatyanarayanaP.V, KesavaiahD.Ch, and Venkataramana.S (2011), "Viscous dissipation and thermal radiation effects on an unsteady MHD convection flow past a semi-infinite vertical permeable moving porous plate," International Journal of Mathematical Archive-2(4), 476-487.

9. Takhar.H.S, Gorla.R.S and Soundalgekar .V.M(1996), "Radiation effects on MHD free convection flow of a radiating gas past a semiinfinite vertical plate,” Int. J. Numerical Methods Heat Fluid Flow, 6, 77-83.

10. Veera Krishna.M (2016), "Unsteady MHD Flow in a Rotating Parallel Plate Channel under the Influence of Pressure Gradient with Hall Current Effects," Journal of Scientific Research and Reports, 10(3), 121.http://dx.doi.org/10.9734/JSRR/2016/24123

11. Veea Krishna.M, and Chand Basha.S (2016), "MHD Free Convection three dimensional flow through a porous medium between two vertical plates," Journal of Mathematics, 12, 88-105. DOI: http://dx.doi.org/10.9790/5728-121288105.

12. Veera Krishna.M, and Prakash.J (2015), "Hall Current Effects on Unsteady MHD Flow in a Rotating Parallel Plate Channel Bounded by Porous Bed on the Lower Half-Darcy Lapwood Model," Open Journal of Fluid Dynamics, 5, 275-294. DOI: http://dx.doi.org/10.4236/ojfd.2015.54029. 